\title{
Noorda blitealis Walker, un ravageur majeur du Moringa au Niger (Lepidoptera, Crambidae)
}

\author{
par Alain RATNADASS ${ }^{*(1)}$, Ousmane ZAKARI-MOUSSA**, Halarou SALHA*, \\ Joël MINET*** \& Amadou Asmaou SEYFOULAYE** \\ * ICRISAT, BP 12404, Niamey, Niger <alain.ratnadass@ cirad.fr> \\ ** Université Abdou Moumouni, Faculté d'Agronomie, B.P.10960, Niamey, Niger \\ *** Muséum national d'Histoire naturelle, département Systématique et Evolution, UMR CNRS 7205, \\ C. P. 50 (Entomologie), 57 rue Cuvier, F - 75231 Paris cedex 05 \\ (1) Adresse actuelle : CIRAD, UPR HortSys, F - 34398 Montpellier
}

Résumé. - La pyrale défoliatrice qui constitue, au Niger, le principal ravageur du Moringa, Moringa oleifera (important légume-feuille), a pu être identifiée, pour la première fois, comme Noorda blitealis Walker, 1859. Des observations originales ont été faites sur la biologie de cet Insecte, permettant d'envisager des méthodes de gestion durable des plantations de moringas.

Summary. - Noorda blitealis Walker, a major pest of horse-radish trees in Niger (Lepidoptera: Crambidae). The leaf-eating caterpillar which is, in Niger, the major pest of Moringa oleifera (an important leaf vegetable) was identified for the first time as Noorda blitealis Walker, 1859. Original observations were made on its biology, opening up new prospects for sustainable management of horse-radish tree plantations.

Keywords. - Moringa oleifera, Noorda blitealis, biology, larval behaviour, Niger.

Bien qu'il y ait été introduit tardivement (peu avant l'indépendance), le Moringa (Moringa oleifera Lam., Moringaceae), arbre originaire de l'Inde (PANDEY et al., 2011), est le légume-feuille traditionnel préféré au Niger, où il contribue largement à l'équilibre nutritionnel des populations locales et y est même exploité, en plantations monospécifiques comme culture de rente (SAINT SAUVEUR \& HARTOUT, 2001).

Le Moringa représente ainsi une option majeure dans le cadre des systèmes de culture promus par l'ICRISAT pour les zones semi-arides d'Afrique de l'Ouest, qu'ils soient fondés sur l'irrigation goutte-à-goutte comme les jardins potagers africains (AMG : PASTERNAK et $a l ., 2006$ ) ou sur des dispositifs d'infiltration des eaux de pluie comme le système de biorécupération des terres dégradées (BDL : PASTERNAK et al., 2009), avec dans ce dernier cas l'espèce voisine, plus résistante à la sécheresse, Moringa stenopetala (Baker f.) Cufod., originaire d'Ethiopie (YISEHAK et al., 2011).

$\mathrm{Si}$, en saison des pluies (de juillet à septembre), le Moringa ne fait pas l'objet d'attaques significatives d'insectes ravageurs, il subit en revanche en saison sèche (le reste de l'année, donc) d'importants dégâts d'une chenille de pyrale défoliatrice causant des pertes considérables (ABASSE et al., 2007). Malgré l'importance des attaques (fig. 1), réduisant les producteurs à appliquer sur le feuillage des insecticides, y compris du DDT dans la région de Maradi (SAINT SAUVEUR \& HARTOUT, 2001 ; ABASSE et al., 2007), cet Insecte n'avait pas été identifié à ce jour, ni fait l'objet d'études en vue de la mise en œuvre de méthodes de lutte durables.

Aussi des études ont-elles été entreprises dans la région de Tillabéri, respectivement en 2007 dans le village de N'Goroua (département de Kollo, $13^{\circ} 22^{\prime} \mathrm{N}, 1^{\circ} 57^{\prime} \mathrm{E}$ ) et en 2009 sur la station ICRISAT de Sadoré (département de Say, $13^{\circ} 15^{\prime} \mathrm{N}, 2^{\circ} 17^{\prime} \mathrm{E}$ ). 

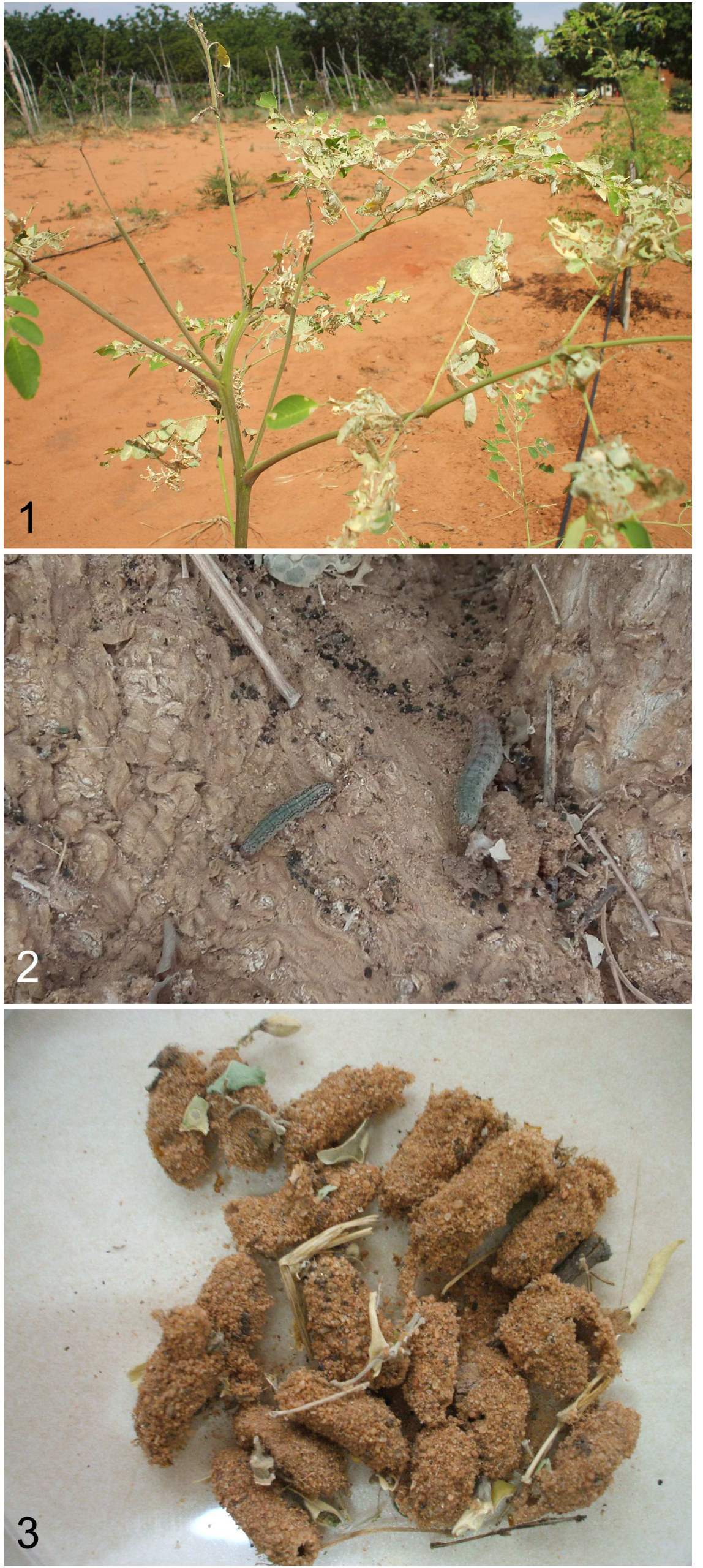

Fig. 1-3. - Noorda blitealis Walker. - 1, Dégâts sur feuillage de Moringa oleifera Lam. - 2, Chenilles au pied d'un M. oleifera. 3 , Cocons.
En novembre 2007, les observations qualitatives effectuées chez trois producteurs de N'Goroua ont montré que l'insecte passait par cinq stades larvaires (L1 à L5) avant de se nymphoser, et qu'on ne trouvait les L1 que dans le feuillage, alors qu'à partir du stade L2, les chenilles passaient la journée dans le sol.

En décembre 2009, des observations effectuées sur quatre plants (moringas) de l'AMG de la station de Sadoré ont montré que, pendant la journée, il y avait autant d'individus immatures de ce ravageur dans le feuillage que dans le sable sous les arbres (respectivement 8,0 et 7,3 par arbre : fig. 2), bien qu'il y eût plus de jeunes stades dans le feuillage (34\% des larves) et plus de stades âgés dans le sable ( $90 \%$ des larves). Aucun cocon n'a été trouvé dans le feuillage, alors que plusieurs cocons ont été retrouvés dans le sable (19 par arbre en moyenne : fig. 3).

Une étude au laboratoire effectuée sur des larves prélevées à N'Goroua en 2007, avec trois types de conditions d'élevage (testés chacun sur dix larves à divers stades) a fourni, au bout de 45 jours, les résultats suivants : (1) absence de nymphose et mortalité de $20 \%$ des larves mises en présence de feuilles seules (sans sable) ; (2) un taux de nymphose de $30 \%$ et une mortalité de $70 \%$ sur sable seul (en l'absence de feuilles) ; (3) un taux de nymphose élevé $(90 \%)$ et une faible mortalité $(10 \%)$ en présence à la fois de sable et de feuilles (cellesci étant invariablement remplacées au moins tous les deux jours). 
Les larves prélevées à Sadoré en 2009 ont également été utilisées dans une expérience de laboratoire (non renouvelée) où l'on a mis en présence de feuilles fraîches et de sable, ou de feuilles seules, ou de sable seul, soit les plus grandes larves (L4 et L5; récoltées sur le feuillage d'une part, dans le sable d'autre part), soit de jeunes larves (L2 ; récoltées sur le feuillage). Les feuilles étaient remplacées tous les deux jours et l'essai a été arrêté au bout d'une semaine, après nymphose ou mort de toutes les larves élevées dans des conditions données.

On a constaté un taux de nymphose maximal et une mortalité minimale quand les larves étaient mises en présence de feuilles fraîches et de sable (qu'il s'agisse de larves âgées, récoltées sur le feuillage ou dans le sable, ou de jeunes larves récoltées sur le feuillage). En revanche, seules les larves âgées se nymphosaient lorsque mises en présence de sable seul, tandis qu'on obtenait un faible taux de nymphose dans le cas des larves, âgées ou jeunes, mises en présence de feuilles seules.

Les chrysalides obtenues ont été conservées au laboratoire jusqu'à émergence des adultes, qui ont été formellement identifiés par le quatrième auteur comme Noorda blitealis Walker, 1859 (Crambidae, Noordinae - voir MINET, 1980), un ravageur important du Moringa en Inde (PATEL et al., 2009a), ainsi qu'en Ethiopie.

Cette identification permet d'envisager l'application des méthodes de lutte préconisées contre ce ravageur en Inde, en privilégiant les applications d'insecticides biologiques (Spinosad, Bacillus thuringiensis) (PATEL et al., 2009b) plutôt que l'utilisation de produits tels que le Carbaryl, le Dichlorvos, le Malathion, le Fenthion ou le Chlorpyrifos - reconnus efficaces, mais moins respectueux de l'environnement - ou le recours à des méthodes physiques peu pratiques (échenillage et piégeage lumineux).

Les observations sur la biologie de Noorda blitealis suggèrent que les chenilles de cette espèce ont besoin à la fois de feuilles et de sable pour compléter leur développement et qu'elles ne se contentent pas de se nymphoser dans le sol, au pied des moringas, mais effectuent (à partir d'un certain âge) des déplacements verticaux circadiens, descendant dans le sable et remontant vers le feuillage, respectivement - semble-t-il - aux heures chaudes de la journée et aux heures plus fraîches de la soirée.

Cela ouvre des perspectives de régulation des populations du ravageur en favorisant la destruction - éventuellement mécanique - de ses stades endogés (chenilles au repos et chrysalides).

REMERCIEMENTS. - Les auteurs remercient la population de N'Goroua et l'équipe du Programme de Diversification des cultures de l'ICRISAT pour leur collaboration.

\section{AUTEURS CITÉS}

Abasse T. A., Gouzayé A., Woltering L. \& PAsternak D., 2007. - The role of indigenous leafy vegetables on daily diet and rural and urban economy of Niger. Acta Horticulturae, 752 : 35-40.

MinET J., 1980. - Création d'une sous-famille particulière pour Noorda Walker, 1859, et définition d'un nouveau genre parmi les Odontiinae [Lep. Pyralidae]. Bulletin de la Société entomologique de France, 85 (3-4) : 79-87.

PANDEy A., PradheEP K., GuPTA R., NAYAR E. R. \& Bhandari D. C., 2011. - 'Drumstick tree' (Moringa oleifera Lam.): a multipurpose potential species in India. Genetic Resources and Crop Evolution, $58(3): 453-460$.

PASternak D., Nikiema A., Senbeto D., Dougbedji F. \& Woltering L., 2006. - Intensification and improvement of marketgardening in the Sudano-Sahel region of Africa. Chronica Horticulturae, 46 (4) : 24-28.

Pasternak D., Senbeto D., Nikiema A., Kumar S., Fatondui D., Woltering L., Ratnadass A. \& NDJEUNGA J., 2009. - Bioreclamation of degraded African lands with women empowerment. Chronica Horticulturae, 49 (2) : 24-27. 
Patel B. P., RADAdia G. G. \& PANDYA H. V., 2009a. - Population dynamics of leaf eating caterpillar, Noorda blitealis Walk. on drumstick, Moringa oleifera L. Insect Environment, 15 (2) : 69-71.

2009b. - Evaluation of insecticides against leaf eating caterpillar, Noorda blitealis Walk. on drumstick, Moringa oleifera L. Insect Environment, 15 (2) : 72-73.

Saint Sauveur A. DE \& HARTOUT G., 2001. - Moringa culture and economy in Niger (p. 29-44). In : Fuglie L. J. (ed.), The miracle tree - the multiple attributes of Moringa. Wageningen, The Netherlands, CTA, $172 \mathrm{p}$.

YiseHAK K., Solomon M. \& TADELle M., 2011. - Contribution of Moringa (Moringa stenopetala, Bac.), a highly nutritious vegetable tree, for food security in South Ethiopia: a review. Asian Journal of applied Sciences, 4 (5) : 477-488. 